\title{
Carcinosarcoma of the uterus: A case report
}

\author{
YASUSHI ADACHI ${ }^{1,2}$, HIROFUMI NONOGAKI $^{3}$, SACHIKO MINAMIGUCHI $^{4}$, \\ MING LI $^{2}$ and SUSUMU IKEHARA ${ }^{2}$
}

\author{
${ }^{1}$ Division of Surgical Pathology, Toyooka Hospital, Toyooka, Hyogo 668-8501; ${ }^{2}$ Department of Stem Cell Disorders, \\ Kansai Medical University, Hirakata, Osaka 573-1010; ${ }^{3}$ Department of Obstetrics and Gynecology, Toyooka Hospital, \\ Toyooka, Hyogo 668-8501; ${ }^{4}$ Department of Diagnostic Pathology, Kyoto University Hospital, Kyoto, Kyoto 606-8507, Japan
}

Received July 3, 2015; Accepted October 16, 2015

DOI: $10.3892 / \mathrm{mco} .2016 .744$

\begin{abstract}
Carcinosarcoma is a rare malignant tumor of the uterus with a poor prognosis. We herein present a case of uterine carcinosarcoma in a 67-year-old woman suffering from pervaginal bleeding for 3 months, with progressive anemia. Upon biopsy of the endometrium, the tumor was diagnosed as endometrioid adenocarcinoma. Total hysterectomy, bilateral salpingo-oophorectomy and pelvic lymph node dissection were performed. The histological examination revealed that the tumor consisted of serous adenocarcinoma and sarcoma of homologous components. After the operation, chemotherapy with paclitaxel and carboplatin was administered. At approximately 3 years postoperatively, the patient remains alive and recurrence-free.
\end{abstract}

\section{Introduction}

Carcinosarcomas of the uterus (malignant mixed Müllerian tumors) are a rare occurrence, accounting for only 2-5\% of all uterine malignancies (1-4). Carcinosarcomas are, however, highly aggressive, and are composed of epithelial and mesenchymal elements (5). Carcinosarcomas are classified into two histological subtypes based on their sarcomatous component, namely homologous or heterologous (6); those of the homologous type tend to be fibrosarcomas, endometrial stromal tumors or leiomyosarcomas, while those of the heterologous type consist of a sarcomatous component made up of tissues that are non-native to the uterus, and include rhabdomyosarcomas, chondrosarcomas, osteosarcomas and liposarcomas. In both types, the carcinomatous component is mainly composed of endometrioid, serous or clear-cell type adenocarcinoma. Homologous and heterologous carcinosarcomas arise with approximately equal frequencies (7).

Correspondence to: Professor Susumu Ikehara, Department of Stem Cell Disorders, Kansai Medical University, 2-5-1 Shinmachi, Hirakata, Osaka 573-1010, Japan

E-mail: ikehara@hirakata.kmu.ac.jp

Key words: uterus, homologous carcinosarcoma, total hysterectomy, salpingo-oophorectomy, chemotherapy
We herein present a case of a homologous carcinosarcoma of the uterus in a 67-year-old woman.

\section{Case report}

A 67-year-old woman, who was gravida 3, para 3, was referred to Toyooka Hospital (Toyooka, Japan) for evaluation of anemia and genital bleeding that had continued for 3 months. The patient was found to be anemic [hemoglobin $(\mathrm{Hb})$ concentration, $7.3 \mathrm{~g} / \mathrm{dl}$ ] at the follow-up blood examination for hyperlipidemia. An upper and lower gastrointestinal endoscopy was performed and failed to identify the source of the bleeding in the gastrointestinal tract. On abdominal ultrasonography, the uterus appeared to be enlarged; a subsequent CT scan revealed the presence of a tumor of the uterine corpus.

On biopsy of the endometrium, the tumor was diagnosed as endometrioid adenocarcinoma. Therefore, total hysterectomy, bilateral salpingo-oophorectomy and pelvic lymph node dissection were performed. There was no macroscopic evidence of intra-abdominal dissemination and/or lymph node involvement. The total weight of the uterus, fallopian tubes and ovaries was $206 \mathrm{~g}$. The enlarged uterus approached the size of the head of a newborn infant (Fig. 1). The tumor per se measured 60x50x50 mm and filled the uterine cavity. The histological examination revealed that the tumor consisted of two components (Fig. 2A-C): One was an adenocarcinoma, exhibiting papillary growth and tubular architecture (Fig. 2B), while the other was a sarcoma containing atypical mesenchymal cells (Fig. 2C). The tumor was mainly an adenocarcinoma, but part of the tumor comprised both adenocarcinomatous and sarcomatous components. Since the adenocarcinoma exhibited a mainly papillary architecture and was positive for p53 (Fig. 2D), it was diagnosed as a moderately differentiated serous adenocarcinoma. The sarcoma was positive for vimentin and CD10, and partially positive for desmin and $\alpha$-smooth muscle actin ( $\alpha$ SMA) (Fig. 3); it was also positive for p53 (Fig. 2D), but did not contain heterologous elements. The tumor was therefore diagnosed as a carcinosarcoma (serous adenocarcinoma with a homologous sarcomatous component). The tumor was limited to the uterine corpus, without metastasis to the lymph nodes. The depth of myometrial invasion of the tumor was $<1 / 2$. Furthermore, there was no evidence of tumor in the ovaries or fallopian tubes (pT1A, pN-0, pMx; stage $1 \mathrm{~A}$ according to the 2008 International Federation 

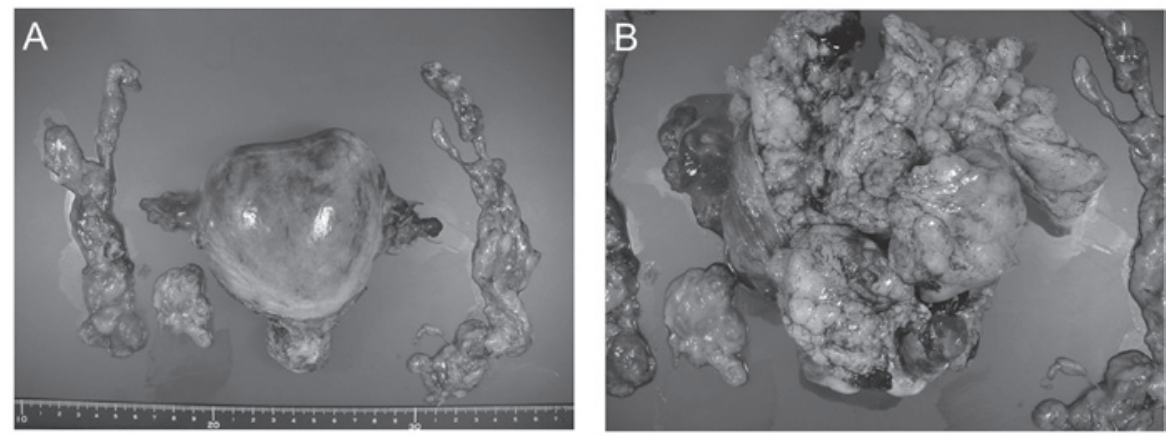

Figure 1. Macroscopic appearance of the resected uterus, fallopian tubes and ovaries. (A) External appearance of the resected surgical specimen and (B) macroscopic appearance of the uterine cavity.
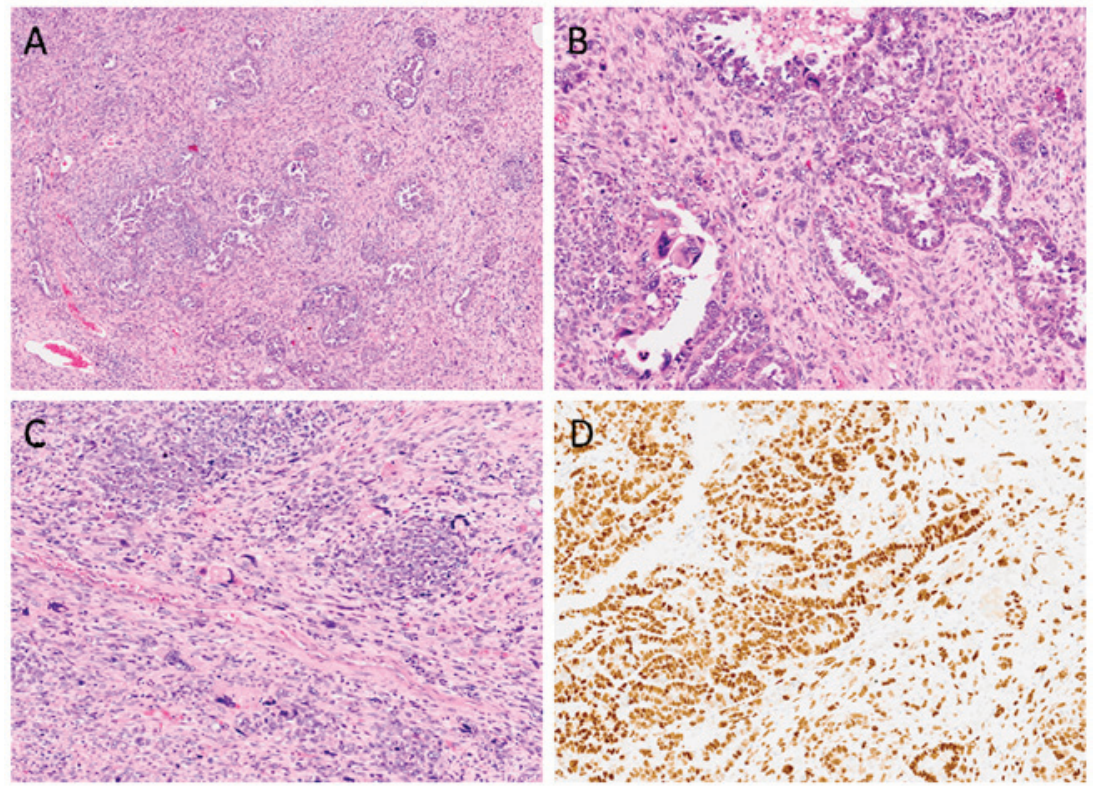

Figure 2. Histological examination of the uterine carcinosarcoma. H\&E staining at a magnification of (A) x4 and (B and C) x10. (D) p53-immunohistochemical staining (magnification, x10). (D) p53-immunohistochemical staining was positive in both the carcinomatous and sarcomatous component (magnification, x10).

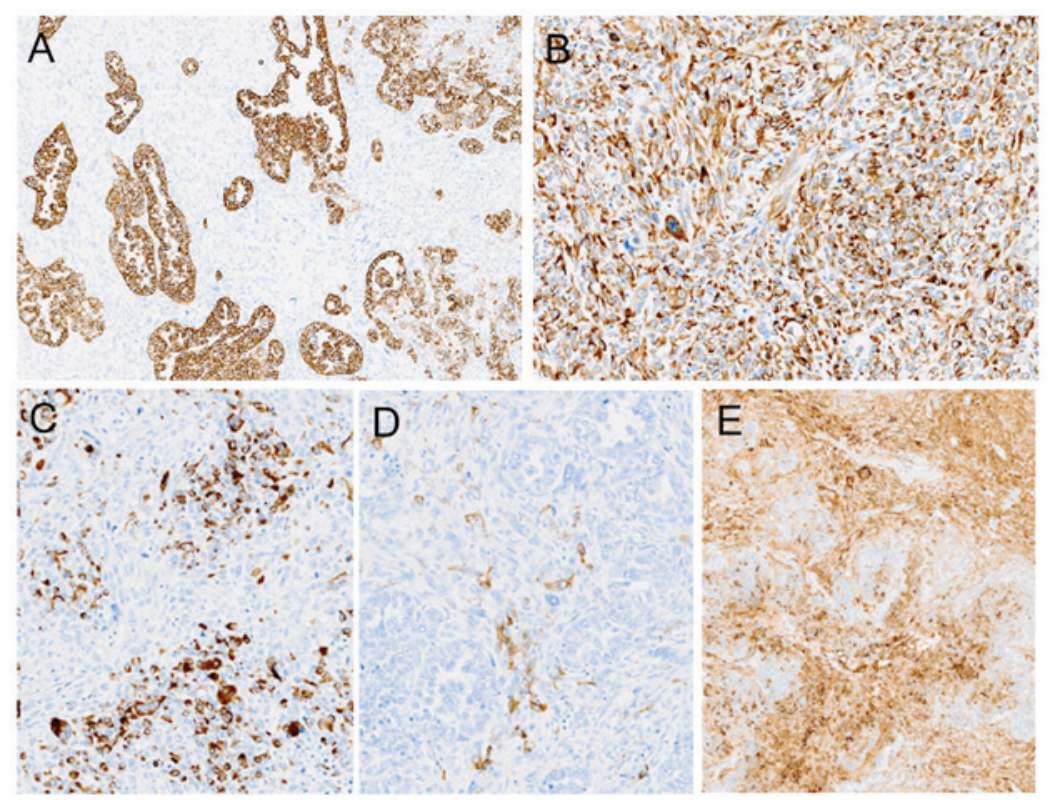

Figure 3. Immunohistochemical analysis of the uterine carcinosarcoma. (A) The adenocarcinomatous component was positive for AE1/AE3. The sarcomatous component was (B) positive for vimentin and partially positive for (C) desmin, (D) $\alpha$-smooth muscle actin and (E) CD10. Magnification, $\mathrm{x} 4$ in (A) and x10 in (B-E). 
of Gynecology and Obstetrics staging criteria). After the operation, chemotherapy with paclitaxel and carboplatin was administered. At $\sim 3$ years postoperatively, the patient remains alive and recurrence-free.

\section{Discussion}

Carcinosarcomas occur mainly in women $\sim 15-17$ years after menopause $(4,8,9)$, with the most common symptoms being genital bleeding and uterine enlargement (10). Abdominal pain also occurs in a proportion of the cases (11). In the present case, the carcinosarcoma was diagnosed 15 years after menopause, with the patient complaining of vaginal bleeding for 3 months prior to the consultation. The Hb levels in the peripheral blood had decreased to $7.3 \mathrm{~g} / \mathrm{dl}$, which was likely due to the genital bleeding. These symptoms and findings were typical of uterine carcinosarcoma, although the patient did not complain of abdominal pain.

Carcinosarcomas are characterized by an aggressive clinical course and an extremely poor prognosis. It has been previously reported that $70-90 \%$ of tumor-related deaths occurred within 18 months after diagnosis $(2,12)$. However, a recent study reported that the prognosis of uterine carcinosacomas had improved, with an overall median survival of 39 months (13).

Uterine carcinosarcomas are mixed epithelial and stromal tumors, with both components being malignant $(6,11)$. Homologous carcinosarcomas have a sarcomatous component of fibrosarcoma, endometrial stromal sarcoma and/or leiomyosarcoma. By contrast, the heterologous type includes sarcomatous components that are made up of tissues non-native to the uterus. The carcinomatous component is mainly composed of endometrioid, serous or clear-cell type adenocarcinoma. In our case, there was a dominant epithelial lesion that displayed papillary growth and, on immunohistological analysis, was p53-positive, suggesting that the epithelial tumor was a serous adenocarcinoma. However, atypical mesenchymal cells were identified in the stroma in the serous adenocarcinoma. These atypical mesenchymal cells were CD10-positive, or $\alpha$ SMA- and desmin-positive, suggesting that the sarcoma in this tumor consisted of homologous components. These results suggest that the carcinosarcoma in our case was a homologous carcinosarcoma.

At approximately 3 years postoperatively, there are no indications of recurrence of the carcinosarcoma. The patient is closely monitored using diagnostic imaging modalities.

\section{Acknowledgements}

The patient provided written informed consent for the publication of her medical details. The present study was approved by the Ethics Committee of Toyooka Hospital. The authors would like to thank Ms. K Ando (Department of Stem Cell Disorders, Kansai Medical University), Ms. K. Miyamura, Ms. S. Kawasaki (Toyooka Hospital) and Mr. Hilary Eastwick-Field for the preparation of this study, and Ms. H. Ogaki, Mr. K. Nagaoka, Mr. T. Kuge and Mr. H. Takenaka (Toyooka Hospital) for their expert technical assistance.

\section{References}

1. Amr SS, Tavassoli FA, Hassan AA, Issa AA and Madanat FF. Mixed mesodermal tumor of the uterus in a 4-year-old girl. Int J Gynecol Pathol 5: 371-378, 1986.

2. Barwick KW and LiVolsi VA: Malignant mixed müllerian tumors of the uterus. A clinicopathologic assessment of 34 cases. Am J Surg Pathol 3: 125-135, 1979.

3. Chuang JT, Van Velden DJ and Graham JB: Carcinosarcoma and mixed mesodermal tumor of the uterine corpus. Review of 49 cases. Obstet Gynecol 35: 769-780, 1970.

4. Williamson EO and Christopherson WM: Malignant mixed mullerian tumors of the uterus. Cancer 29: 585-592, 1972.

5. Sebenik M, Yan Z, Khalbuss WE and Mittal K: Malignant mixed mullerian tumor of the vagina: Case report with review of the literature, immunohistochemical study and evaluation for human papilloma virus. Hum Pathol 38: 1282-1288, 2007.

6. Jin Z, Ogata S, Tamura G, Katayama Y, Fukase M, Yajima M and Motoyama T: Carcinosarcomas (malignant mullerian mixed tumors) of the uterus and ovary: A genetic study with special reference to histogenesis. Int J Gynecol Pathol 22: 368-373, 2003.

7. Spaziani E, Picchio M, Petrozza V, Briganti M, Ceci F, Di Filippo A, Sardella B, De Angelis F, Della Rocca C and Stagnitti F: Carcinosarcoma of the uterus: A case report and review of the literature. Eur J Gynaecol Oncol 29: 531-534, 2008.

8. Macasaet MA, Waxman M, Fruchter RG, Boyce J, Hong P, Nicastri AD and Remy JC: Prognostic factors in malignant mesodermal (mullerian) mixed tumors of the uterus. Gynecol Oncol 20: 32-42, 1985.

9. Gallup DG, Gable DS, Talledo OE and Otken LB Jr: A clinical-pathologic study of mixed mullerian tumors of the uterus over a 16-year period-the medical college of georgia experience. Am J Obstet Gynecol 161: 533-538; Discussion 538-539, 1989.

10. Ali S and Wells M: Mixed mullerian tumors of the uterine corpus: A review. Int J Gynecol Cancer 3: 1-11, 1993.

11. Villena-Heinsen C, Diesing D, Fischer D, Griesinger G, Maas N, Diedrich K and Friedrich M: Carcinosarcomas - a retrospective analysis of 21 patients. Anticancer Res 26: 4817-4823, 2006.

12. Norris HJ, Roth E and Taylor HB: Mesenchymal tumors of the uterus. II. A clinical and pathologic study of 31 mixed mesodermal tumors. Obstet Gynecol 28: 57-63, 1966.

13. Rauh-Hain JA, Shoni M, Schorge JO, Goodman A, Horowitz NS and del Carmen MG: Prognostic determinants in patients with uterine and ovarian carcinosarcoma. J Reprod Med 58: 297-304, 2013. 